Neurosurg Focus 23 (3):E4, 2007

\title{
Cyclic Cushing syndrome: definitions and treatment implications
}

\author{
Dennis A. Velez, M.D., Marc R. Mayberg, M.D., and William H. Ludlam, M.D., Ph.D. \\ Seattle Neuroendocrine Program, Swedish Neuroscience Institute, Seattle, Washington
}

\begin{abstract}
$\checkmark$ Endogenous Cushing syndrome (CS) results from hypercortisolemia caused by excess adrenocorticotropic hormone production in a pituitary adenoma or ectopic tumor, or by an adrenal tumor that directly produces excess cortisol. The diagnosis can usually be ascertained with a reasonable degree of certainty based on clinical and laboratory findings of hypercortisolism. There are patients, however, in whom the production of excess cortisol exhibits a cyclic or intermittent pattern, and, as a result, the clinical symptoms may be quite complex and varied. In these patients the hypothalamic-pituitary-adrenal axis may be normal between cycles, and dexamethasone suppression testing may produce a paradoxical response. In the present article, the authors provide a definition of cyclic Cushing syndrome, review the causes and its potential pathophysiological mechanisms, and discuss the treatment options based on a review of the available literature. (DOI: 10.3171/FOC-07/09/E4)
\end{abstract}

\section{Key WoRdS • cyclic Cushing disease - hypercortisolism • pituitary adenoma • adrenocorticotropic hormone}

$\mathrm{T}$ HE pathological or iatrogenic state of glucocorticoid excess combined with its clinical and biochemical findings is known as CS. Endogenous CS results from prolonged exposure to excess glucocorticoids produced by the adrenal cortex or an adrenal tumor. To understand the causes of CS it is appropriate to classify the hypercortisolemic state by its dependence on corticotropin (ACTH). Pituitary corticotrophic adenomas account for about 80 to $90 \%$ of all noniatrogenic adult cases of CS. Cortisol-producing adrenal as well as non-pituitary ACTHsecreting lesions such as lung carcinoid, small cell lung carcinoma, pheochromocytoma, medullary thyroid carcinoma, pancreatic islet tumors, and rare ovarian tumors account for the majority of the balance. Ectopic CRH-producing lesions such as hypothalamic gangliocytomas are exceedingly rare.

A subset of patients with CS presents with variable hormonogenesis, which may or may not exhibit overt clinical expression. During the nadir of hormonogenesis, serum hormone levels may be elevated, normal, or subnormal. The variability of these cycles may be regular or irregular.

\section{Definition of Cyclic CS}

Although not identified in most large series of patients with CS, the presence of cyclic CS is being increasingly

Abbreviations used in this paper: $\mathrm{ACTH}=$ adrenocorticotropic hormone; $\mathrm{CRH}=$ corticotropin-releasing hormone; $\mathrm{CS}=$ Cushing syndrome; TSA = transsphenoidal adenomectomy; UFC = urinary free cortisol. recognized as a clinical entity. ${ }^{18}$ Its diagnosis, although challenging, can be made by demonstrating three peaks and two troughs of cortisol production. ${ }^{2}$ There are no available data to date that define what minimal biochemical threshold should be used to establish the diagnosis of cyclic CS. In general, however, the tests results used to document episodes of hypercortisolemia in cyclic CS will be similar to those of traditional CS except one will observe periodic episodes of "normal" test results. The duration of the cycles has been described as being 12 hours to 86 days. This range is in contrast to the condition referred to as intermittent CS, in which the cycle of cortisol production is irregular and ranges from 10 episodes of relapse in 3 months to two episodes of exacerbation in 5 years. The term CS with periodic hormonogenesis has been proposed to unify these conditions. Implicit in the former condition is the expectation of a relatively predictable pattern of cortisol production, with similar distance between the peaks.

\section{Clinical Presentation of Cyclic CS}

In patients with CS, excessive cortisol production is responsible for the clinical phenotype of central obesity with supraclavicular fat accumulation, purple striae, proximal muscle weakness, hypertension, diabetes mellitus, and neuropsychiatric disturbances, among other findings. In patients with cyclic CS, signs and symptoms may be limited to a few subtle clinical clues such as recurrent peripheral edema, cardiac rhythm disturbances, or hypokalemia. Shapiro and Shenkman, ${ }^{16}$ in their experience in four cases and review of 44 cases, divided cases into five general cat- 
egories based on the periodicity of the symptoms and the clinical manifestations. It is interesting that, in their review, a group of patients exhibited variable clinical expression and a variable temporal course.

\section{Origin of Cyclic CS}

Although cyclic CS can be caused by a corticotrophic adenoma of the pituitary, as these comprise about $80 \%$ of the cases of ACTH-dependent CS, it has also been reported in association with adrenal hyperplasia and adenomas as well as in association with malignant carcinoid tumor of the lung, ${ }^{6}$ pheochromocytoma, ${ }^{17}$ bronchial adenoma, ${ }^{15}$ and oncocytic carcinoid of the kidney. ${ }^{8}$ Adrenal masses and adrenocortical nodular dysplasia have also been reported.,16 The association with confirmed hypothalamic disorders was reported by Shapiro and Shenkman. ${ }^{16}$ Although cyclic CS has been historically thought to be a very rare entity, it is now being recognized with increasing frequency, which may be due, in part, to increased consideration of and testing for the disease. Further confounding the ability to determine the incidence, ACTH staining and Crooke hyaline changes may be negative in tumor tissue in patients with cyclic CS because of the absence of persistently elevated blood ACTH and cortisol levels. This makes the retrospective recognition of the disease in surgical series very difficult.

\section{Diagnosis of Cyclic CS}

The diagnosis of cyclic CS presents a challenge both from a clinical and laboratory evaluation perspective. Although no specific diagnostic study will differentiate cyclic CS from regular CS, clinical evaluation involving special attention to subtle signs and symptoms of hypercortisolism is imperative to proceed with laboratory evaluation. Laboratory testing can be timed to correlate with signs and symptoms of episodes of hypercortisolemia. Standard measurement of 24-hour UFC has a sensitivity of 95 to $100 \%$ and specificity of 94 to $98 \%$ for the detection of CS, when conducted with high-performance liquid chromatography. ${ }^{12}$ Ideally three 24-hour UFC samples would be obtained to increase the diagnostic yield because there is variability in cortisol secretion from day to day. A midnight plasma cortisol level with values greater than $207 \mathrm{nmol} / \mathrm{L}$ has a sensitivity of $96 \%$ and a specificity of $100 \%$ in differentiating CS from pseudo-Cushing states. ${ }^{13}$ Salivary cortisol samples obtained at 11 p.m. have a sensitivity of $92 \%$ and a specificity of $95 \% .{ }^{11}$ Cortisol levels in saliva have a high correlation with free serum cortisol and are stable for up to 1 week at room temperature once collected. Dexamethasone suppression testing, when carefully done and interpreted, also plays a significant role in the confirmation of the diagnosis of CS.

In patients suspected of having cyclic CS, significant clinical clues of periodic hypercortsolism are gathered from the history and from subtle clinical findings. Paradoxical responses to dexamethasone suppression testing are usually a consistent laboratory finding. Brown and colleagues ${ }^{5}$ described the case of a patient with a pituitary adenoma in whom there was a paradoxical increase in cortisol production before the first dose of dexamethasone was administered. Subsequently Atkinson and coworkers ${ }^{1}$ described a
D. A. Velez, M. R. Mayberg, and W. H. Ludlam

pituitary adenoma in a case in whom both cortisol and ACTH patterns of secretion followed different rhythms. Pituitary function is normal between cycles and imaging diagnostic studies such as pituitary magnetic resonance imaging, adrenal scanning, and inferior petrosal sinus sampling are often negative. Yamamoto et al..$^{20}$ described two cases in which there was positive bilateral inferior petrosal sinus sampling; the patients underwent transsphenoidal exploration of the pituitary and no tumor was found. Both patients eventually were found to harbor an ACTH-secreting lung lesion. The authors indicated that CRH was used to increase the sensitivity and specificity of the inferior petrosal sinus sampling. They concluded that the likely reason for the false-positive finding was the presence of intermittent CS.

Ultimately a long period of surveillance as well as careful interpretation and reevaluation of clinical and laboratory findings may be needed to demonstrate the cyclic nature of CS. Salivary cortisol samples collected over time in an outpatient setting are a highly efficacious, noninvasive way of establishing the diagnosis of cyclic CS. ${ }^{11}$ The diagnosis requires a high index of suspicion not only in the aforementioned situations but also in patients who undergo selective TSA for presumed CS and in whom the cushingoid features are slow to resolve or in whom signs and symptoms of the disorder return after uncomplicated operative interventions. Clinicians trying to confirm or exclude a diagnosis of cyclic CS must perform repeated cycles of tests for hypercortisolemia and be comfortable with the fact that some test results may be normal in the presence of the disease during periods of diminished tumoral activity.

Although it can be very useful to look for three peaks and two troughs of cortisol production in patients being evaluated for cyclic CS, ${ }^{2}$ it is ultimately the level of clinical suspicion for the disease that drives the decision for how much testing is ultimately performed. We suggest that an effective preliminary screening protocol is to obtain a series of four 24-hour UFC collections, midnight salivary cortisol collections, and blood draws for cortisol and ACTH during periods of symptomatic hypercortisolemia (that is, during times of increasing central weight, edema, hirsutism, acne, acid reflux, and facial redness). Negative results for four sets of these tests (performed at times consistent with clinical hypercortisolemia) largely rules out the presence of cyclic CS, unless clinical suspicion remains high. By contrast, intermittently positive test results for hypercortisolemia may suggest the presence of cyclic CS, and more extensive testing may be warranted.

\section{Pathophysiological Mechanisms of Cyclic CS and Treatment}

The definitive mechanism responsible for cyclic CS remains unknown. Halberg and colleagues ${ }^{7}$ have shown cycles of steroid production in normal individuals; thus, episodic hypercortisolism may be an exaggeration of the normal cyclical variation in a subgroup of patients. Periodic ACTH secretion has been associated with a cyclic change in central dopaminergic tone. The response to both low and high doses of bromocriptine, a known specific activator of dopaminergic receptors in the central nervous system, including the hypothalamus, has led some investi- 
gators to propose that the hypothalamic dysfunction plays a central role in the development of this condition. ${ }^{18,19}$ The authors of in vitro studies, however, have shown that bromocriptine can also act directly on pituitary adenoma cells and inhibit the release of ACTH. Beckers et al. ${ }^{3}$ have described the case of a patient with cyclic CS who initially underwent bromocriptine therapy for 2 years and subsequently underwent a TSA. Although symptoms rapidly resolved, they returned within 2 months. Treatment with sodium valproate was then instituted, and a prompt normalization of clinical symptoms and laboratory abnormalities was observed, although the rhythmic excretion of urinary cortisol continued. Sodium valproate is a well-known antiepileptic drug that increases $\gamma$-aminobutyric acid levels, which in turn inhibit CRH secretion. It has been reportedly used in CS and in cases of Nelson syndrome. ${ }^{9}$ Contrary to the effects of bromocriptine, the response of ACTH-producing cells to sodium valproate has not been demonstrated. Cyproheptadine, a well-known serotonin antagonist, has been used in some patients with CS and may be helpful in patients with cyclic CS. The high rate of recurrent symptoms after TSA, in combination with the aforementioned observations, implies that hypothalamic dysfunction may play a central role in the pathophysiology of this condition. ${ }^{10}$ The role of TSA in patients with an unequivocal diagnosis of cyclic CS remains uncertain. Beckers et $\mathrm{al}^{3}{ }^{3}$ and Shapiro et al. ${ }^{15}$ have reported that patients with cyclic CS suffer symptom recurrence after TSA. Based on these reports, TSA cannot be recommended as definitive treatment. Prospective studies of patients with a clear-cut diagnosis of cyclic CS will be needed to better define the role of TSA in this setting.

\section{Conclusions}

Cyclic CS has varied clinical presentations and presents a diagnostic challenge. Recurrent, sporadic signs and symptoms of hypercortisolism with normal or fluctuating cortisol values and normal pituitary function, as well as a paradoxical response to dexamethasone suppression testing, are valuable clinical clues that should prompt one to proceed with dynamic investigation during periods of hypercortisolism. Performing a series of four 24-hour UFC collections, midnight salivary cortisol collections, and blood draws for cortisol and ACTH during periods of symptomatic hypercortisolemia can be a very effective initial screening protocol. Based on the available literature, it is not uncommon for these patients to undergo TSA even before the diagnosis is clear. Medical treatments have only shown limited efficacy to date. Studying the clonality of pituitary cells removed during TSA in patients with cyclic CS may shed some light on the pathophysiology of this rare condition. Clinicians who commonly treat patients with pituitary disorders need to be aware of this condition along with its diagnostic pitfalls.

\section{References}

1. Atkinson AB, Chestnutt A, Crothers E, Woods R, Weaver JA, Kennedy L, et al: Cyclical Cushing's disease: two distinct rhythms in a patient with a basophil adenoma. J Clin Endocrinol Metab 60:320-332, 1985

2. Atkinson AB, Kennedy AL, Carson DJ, Hadden DR, Weaver JA,
Sheridan B: Five cases of cyclical Cushing's syndrome. Br Med J (Clin Res Ed) 291:1453-1457, 1985

3. Beckers A, Stevenaert A, Pirens G, Flandroy P, Sulon J, Hennen G: Cyclical Cushing's disease and its successful control under sodium valproate. J Endocrinol Invest 13:923-929, 1982

4. Blau N, Miller WE, Miller ER Jr, Cervi-Skinner SJ: Spontaneous remission of Cushing's syndrome in a patient with an adrenal adenoma. J Clin Endocrinol Metab 40:659-663, 1975

5. Brown RD, Van Loon GR, Orth DN, Liddle GW: Cushing's disease with periodic hormonogenesis: one explanation for paradoxical response to dexamethasone. J Clin Endocrinol Metab 36: 445-451, 1973

6. Frenia D: Cyclic Cushings syndrome caused by periodic secretion of ACTH from a bronchial carcinoid tumor. Chest:124: 304, 2003

7. Halberg F, Engeli M, Hamburger C: The 17-ketosteroid excretion of a healthy man on weekdays and weekends. Exp Med Surg 23:61-69, 1965

8. Hannah J, Lippe B, Lai-Goldman M, Bhuta S: Oncocytic carcinoid of the kidney associated with periodic Cushing's syndrome. Cancer 61:2136-2140, 1988

9. Lamberts SW, Verleun T, Bons EG, Uitterlinden P, Oosterom R: Effect of cyproheptadine, desmethylcyproheptadine, gamma-amino-butyric acid and sodium valproate on adrenocorticotrophin secretion by cultured pituitary tumor cells from three patients with Nelson's syndrome. J Endocrinol 96:401, 1983

10. Mantero F, Scaroni CM, Albiger NM: Cyclic Cushing's syndrome: an overview. Pituitary 7:203-207, 2004

11. Mosnier-Pudar H, Thomopoulos P, Bertagna X, Fournier C, Guiban D, Luton JP: Long-distance and long-term follow-up of a patient with intermittent Cushing's disease by salivary cortisol measurements. Eur J Edocrinol 133:313-316, 1995

12. Newell-Price J, Trainer P, Besser M, Grossman A: The diagnosis and differential diagnosis of Cushing's syndrome and pseudoCushing's states. Endocr Rev 19:647-672, 1998

13. Papanicolau DA, Yanovski JA, Cutler GB Jr, Chrousos GP, Nieman LK: A single midnight serum cortisol measurement distinguishes Cushing's syndrome from pseudo-Cushing states. J Clin Endocrinol Metab 83:1163-1167, 1998

14. Raff H, Raff JL, Findling JW: Late-night salivary cortisol as a screening test for Cushing's syndrome. J Clin Endocrinol Metab 83:2681-2686, 1998

15. Shapiro MS, Gutman A, Bruderman I, Myers B, Griffel WB: Cushing's syndrome associated with a bronchial adenoma. Possible periodic hormonogenesis. Isr J Med Sci 11:9191-924, 1975

16. Shapiro MS, Shenkman L: Variable hormonogenesis in Cushing's syndrome. Q J Med 79:351-363, 1991

17. Terzolo M, Alì A, Pia A, Bollito E, Reimondo G, Paccotti P, et al: Cyclic Cushing's syndrome due to ectopic ACTH secretion by an adrenal pheochromocytoma. J Endocrinol Invest 17:869-874, 1994

18. Van Cauter E, Retetoff S: Evidence for two subtypes of Cushing's disease on the analysis of episodic cortisol secretion. N Engl J Med 312:1343-1349, 1985

19. Watanobe H, Aoki R, Takebe K, Nakazono M, Kudo M: In vivo and in vitro studies in a patient with cyclical Cushing's disease showing some responsiveness to bromocriptine. Horm Res 36: 227-234, 1991

20. Yamamoto Y, Davis DH, Nippoldt TB, Young WF Jr, Huston J III, Parisi JE: False-positive inferior petrosal sinus sampling in the diagnosis of Cushing's disease. Report of two cases. J Neurosurg 83:1087-1091, 1995

Manuscript submitted June 14, 2007.

Accepted July 31, 2007.

Address correspondence to: Marc R. Mayberg, M.D., 550 17th Avenue, Suite 500, Seattle, Washington 98122. email: marc. mayberg@swedish.org. 